\title{
Transfer Barrier and Timed Transfer System Application Analysis
}

\author{
Liao Chen ${ }^{1,}$, Xifu Wang ${ }^{1, b}$ \\ ${ }^{1}$ School of Traffic and Transportation, BeiJing JiaoTong University, BeiJing 100044, China; \\ aykcl001@163.com, bxfwang1@bjtu.edu.cn
}

Keywords: Transit network, Transfer barrier, Timed transfer system(TTS), Application.

\begin{abstract}
Under the circumstance of social development, with the rapid growth in passenger travel demand, the demand of development of public transport networks for the urban travel is daily increasing. The service capability of public transport network can't satisfy that all the passengers are able to arrive at destinations directly. In response to these issues, this paper makes research: On one hand, learn about current problems in transit, study the factors that affect the transfer barrier, and evaluate if the bus travel are reasonable. On the other hand, use TTS to guarantee that passengers can enjoy a comfortable and convenient travel experience in the transit area of low frequencies.
\end{abstract}

\section{Introduction}

Under the current environment of social development, passenger travel demand is rapidly growing, with increasing motorization requirements. Passenger travel demand requires adequate supplies. City travel demand increases the demand for development of public transport networks. However, under the present conditions, existing public transport infrastructure in large and medium cities in China generally lags far behind the level of urban traffic of other cities in developed countries in Europe and America. Therefore, we need to design a good bus network, which has a good circuit design, improved station facilities, easy walking paths and coordinating schedules to make the change more powerful and convenient, reducing the transfer barrier.

For bus schedules, Hurdle (1973) [1] takes the operating costs and passenger travel costs minimizing as the objective function, solving the model to obtain the optimized conventional bus stop locations and departure intervals. Kyte et al [2] presents a public transit network based on a main line and passenger traffic hubs, which could facilitate the coordination number of bus in the hub site by identifying the integral hour departure interval. Ceder [3] establishes a single integer optimization model of the bus schedule with the goal of maximizing synchronous arrival transit time, and designed an effective heuristic algorithm applied in sample calculation of two or multiple lines of synchronization. However, he still just considered the simultaneous optimization method for a single period.

Tao Jiang [4] uses heuristic algorithms to study the ground conventional public transit network optimization design. Shuai Dai et al [5] studies the transit time reliability, points out the problems of the current transfer system, and proposes a corresponding mathematical model to optimize. Jianxin Yan et al [6] takes a study on bus dispatching which based on transfer optimization, puts out an analysis of optimal operation of transfer between bus routes and bus area, and studies the corresponding model. Qin Shi et al [7] established a optimization model on the basis of the researches of Xuemei Zhou and Ceder, which takes maximizing the vehicles meeting times as first target, and maximizing the opportunity of multiple vehicles meeting at stops as second target, considering the constraint of the range of first and last bus departure time, the departure interval requirements, and the vehicle amounts of the station holds simultaneously. They select using heuristic algorithm on model solution, and get the schedule of maximum timed transfer opportunity.

Giving priority to the development of public transport have become urban planning of future development trends and objectives. This paper is to do a research on a various qualitative and quantitative analysis about the public transport network optimization, mainly related to research of transfer barrier, timed transfer system (TTS) research and application. 


\section{Transfer Barrier Application Analysis}

Transfer Barrier Function. Passenger travel methods which chosen in the transit services usually depend on the itinerary of transfer options, travel time, ease of transfer and travel cost et al. Therefore, the transfer barrier becomes one of the evaluation factors which determines whether the optimization of transfer impedance transfer service is successful, bus line arrangement is reasonable, or vehicle frequency is appropriate.

Currently in passenger travel that we know of, most of the passenger services can only accept one or up to two times of transfer. If still adding transfer times, it will greatly affect the travel time for passengers, even delays.

Fig.1 shows the passenger travel mode of only needing once transference. The figure shows that, the first line is recorded as line $F$, and the second transfer line is recorded as line $G$. The transfer barrier function can be calculated based on the following steps:

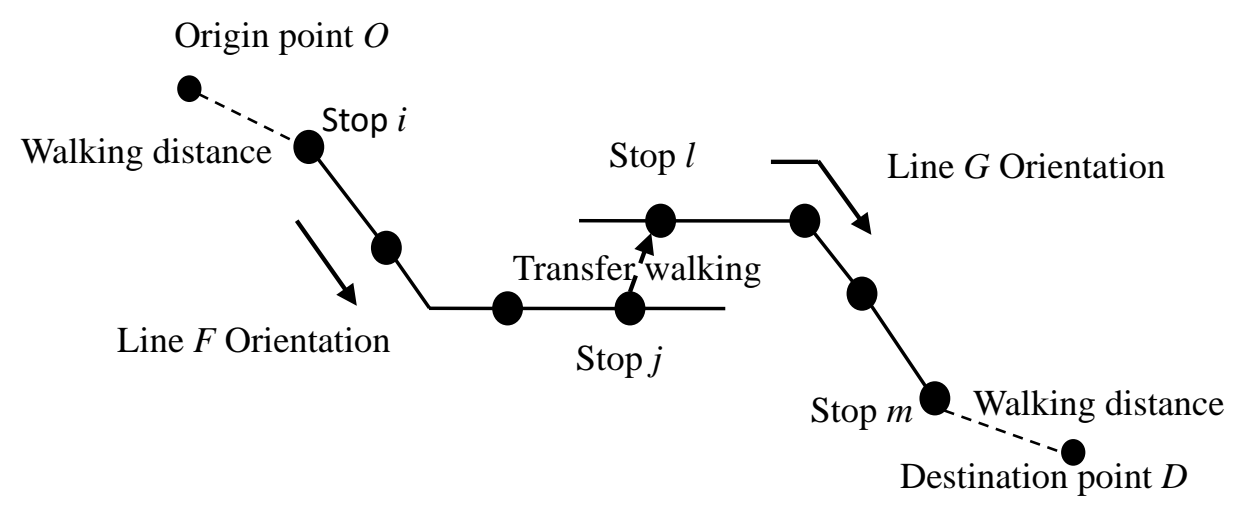

Fig. 1 Travel to destination through one time of transfer

The travel barrier factor of line $G$ could be calculated by Function(1):

$$
t_{j d}=\alpha t_{b}^{G}+\beta h_{j l}+t_{w l}^{G}+t_{l m}^{G}+t_{p}^{G}+t_{s}^{G}+t_{r}^{G}
$$

The travel barrier factor of line $F$ could be calculated by Function(2):

$$
t_{o j}=\alpha t_{b}^{F}+t_{w i}^{F}+t_{i j}^{F}+t_{p}^{F}+t_{s}^{F}+t_{r}^{F}
$$

Therefore, the final travel barrier factor function could be formulated as:

$$
t_{o d}=t_{o j}+t_{j d}
$$

$t_{j d}=$ the travel barrier factor of Line $G$; $t_{o j}=$ the travel barrier factor of Line $F$; $t_{b}=$ the time cost of walking; $h_{j l}=$ the time cost of transfer walking between stop $j$ and stop $l ; t_{w l}=$ the waiting time; $t_{i j}^{F}, t_{l m}^{G}=$ the bus travel time of the line; $t_{p}=$ the fare quantified to the time factor; $t_{s}=$ the comfort level quantified to the time factor; $t_{r}=$ the reliability quantified to the time factor; $\alpha=$ walking penalty factor; $\beta=$ transfer penalty factor.

Numerical Analysis. The sample takes Hebei University of Technology in Tianjin as origin and TianJin Railway Station as destination. In this travel, the commuters are mostly college students. According to the options students take, there are two solutions most likely to be chosen.

Solution 1. Take a bus of Line 649 or 319 in campus, and get off at Liuyuan Station to transfer for the first time. Take a subway train of Tianjin Metro of Line 1, then get off at Xinanjiao Station to transfer for the second time to Tianjin Metro of Line 2, and finally arrive at TianJin Railway Station. 


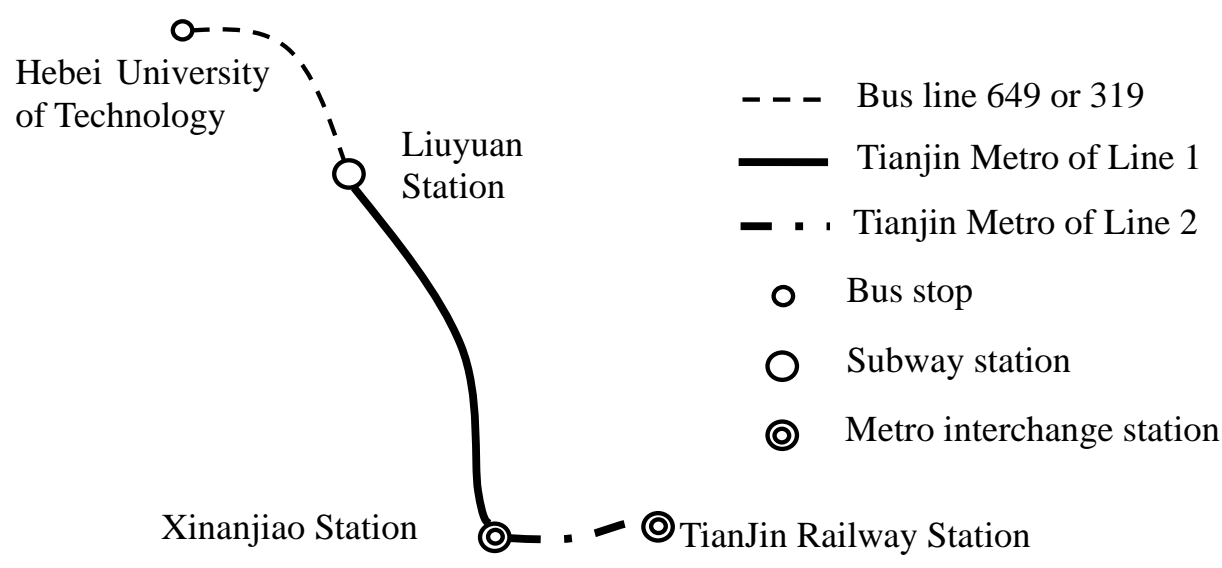

Fig. 2 Travel solution 1

Solution 2. Take a bus of Line 649 in campus, and get off at line terminal Dingzigu Station to transfer to Bus Line 5, and finally arrive at TianJin Railway Station.

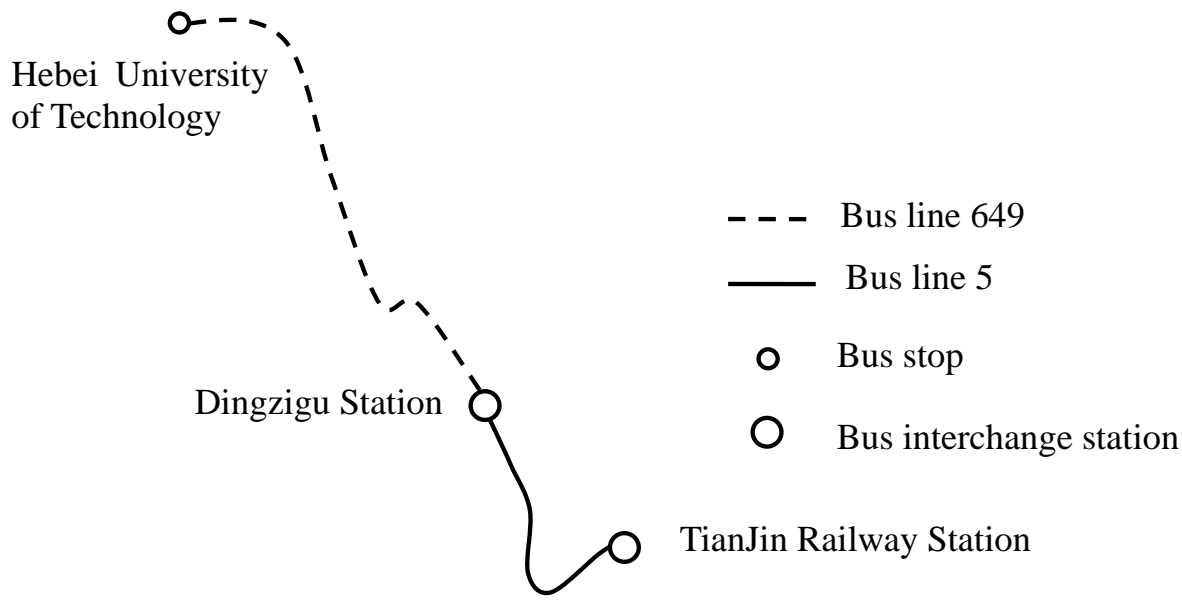

Fig. 3 Travel solution 2

According to the two solutions, students choose to ride the first bus line from school, which could consider walking time and waiting time of Solution 1 are the same as Solution 2 without the need to compare. And according to the characteristics of most of the passengers are college students, comfort of the students on the bus are not necessarily important. So we can think of the comfort as the same impedance in the function. Therefore, the barrier change factors in the function turns into bus travel time, walking time, waiting time, the reliability quantified to the time factor and the fare quantified to the time factor.

Solution 1 transfer barrier function calculation as follows:

(1)the travel time of taking bus of 649 or 319 from school to Liu Yuan Metro station;

(2)the walking time from Liuyuan bus stop to Liuyuan Metro station;

(3)the waiting time in Liuyuan Metro station;

(4)the travel time on Metro Line 1 from Liuyuan Metro station to Xinanjiao station;

(5)the walking time at Xinanjiao station from Metro Line 1 transfer to Metro Line2;

6)the waiting time in Liuyuan Metro station;

(7)the travel time on Metro Line 2 from Xinanjiao station to TianJin Railway Station;

(8)Running time reliability to the time factor, due to regular buses delays for the reason of traffic situation, set the reliability factor of Line 649 as $r_{1}$; subway lines run more reliable, thus set the reliability factor of metro as $r_{2}=1$. The reliability factor of Solution 1 is: $t_{r}=24 r_{1}+28 \mathrm{~min}$

(9)the fare of Line 649 is $¥ 2$, the fare of metro is $¥ 4$. $M=$ Per capita annual income. Set the $M$ of college students as 15000 . The fare quantified of Solution 1 as follows: 


$$
t_{p}=\frac{\sum_{p \in n} y_{p} \bullet d \bullet 480}{M}=\frac{(2+4) \times 260 \times 480}{15000}=49.92 \mathrm{~min}
$$

Summarizing the above calculations, the transfer barrier factor of Solution 1 as following:

$$
t_{O D}^{1}=t_{x l}^{F}+t_{l w}^{G}+t_{l j}^{G}+\beta\left(h_{l s}+h_{h s}\right)+t_{j w}^{H}+t_{j t}^{H}+t_{r}+t_{p}=24 r_{1}+141.87 \mathrm{~min}
$$

Solution 2 transfer barrier function calculation as follows:

(1)the travel time of taking bus of 649 from school to Dingzigu station;

(2)Line 649 shares Dingzigu Station with Line 5, set the walking time as 0;

(3)the waiting time for the bus of Line 5;

(4)the travel time on the bus of Line 5 from Dingzigu station to TianJin Railway Station;

(5)Running time reliability to the time factor, set the reliability factor of Line 649 as $r_{1}$; set the reliability factor of Line 5 as $r_{3}$. The reliability factor of Solution 2 is: $t_{r}=46 r_{1}+42 r_{3}$ min

(6)the fare of Line 649 is $¥ 2$, the fare of metro is $¥ 2$. The fare quantified of Solution 2 as follows:

$$
t_{p}=\frac{\sum_{p \in n} y_{p} \bullet d \bullet 480}{M}=\frac{(2+2) \times 260 \times 480}{15000}=33.3 \mathrm{~min}
$$

Summarizing the above calculations, the transfer barrier factor of Solution 2 as following:

$$
t_{O D}^{2}=t_{x d}^{F}+t_{d w}^{G}+t_{d t}^{G}+t_{r}+t_{p}=46 r_{1}+42 r_{3}+126.3 \mathrm{~min}
$$

Due to the delays of regular buses, time reliability factor $r_{i j}$ is usually bigger than 1 . Thus, according to the results of equation (5) and (7), the transfer barrier of Solution 2 is much bigger than Solution 1. Therefore, we know that, between the pair of OD Hebei University of Technology and Tianjin Railway Station, passengers are usually travel by Solution 1. This result is also consistent with actual passenger travel choice.

\section{Timed Transfer System(TTS) Application Analysis}

TTS Application Fields Analysis. TTS is often used in the transfer type of long departure intervals between bus lines while the departing time interval is the same and synchronous among the lines. TTS requires the line schedules to adapt to the design of public transit network and coordination among existed lines, in order to provide transfer integration service. TTS is usually used in the network of high degree of bus timetable accuracy.

(1) Applied in suburban lines.

Due to the radial space layout between the outskirts of the city and towns, suburban bus routes are generally in radial formation. The characteristics of suburban bus transit network are similar to the network suitable for TTS, which is called Hub and spoke network [9].

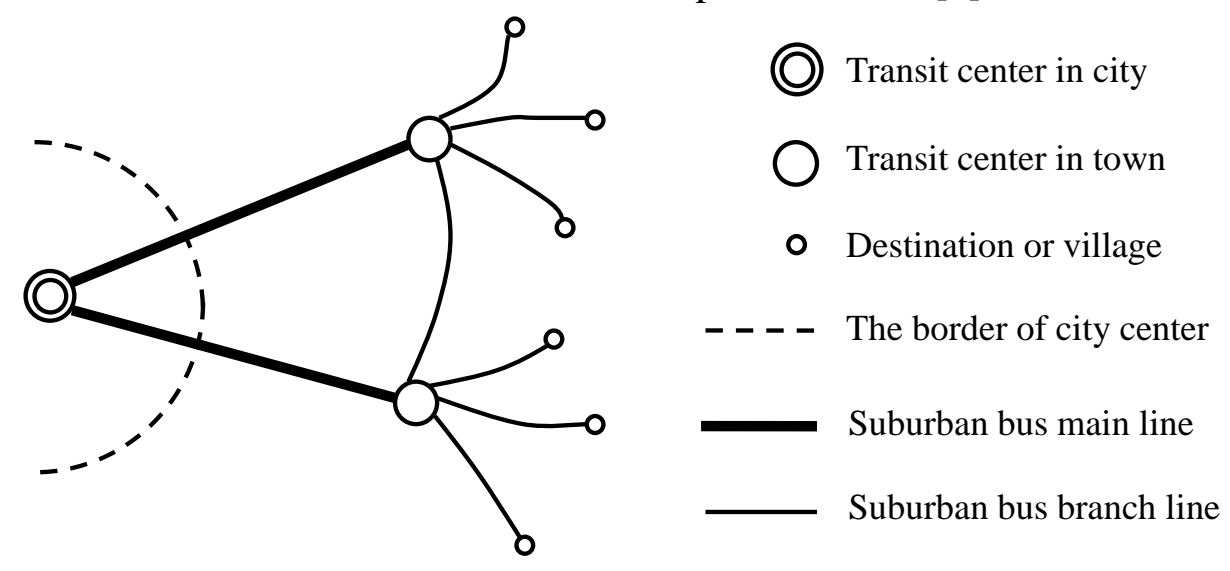

Fig. 4 Hub and spoke network 
(1) Applied in BRT.

Bus Rapid Transit (BRT) is a new bus system between Rapid Rail Transit (RRT) and Normal Bus Transit (NBT), which is a kind of medium-heavy road traffic. BRT does have the quality of rail service and flexibility of bus service, with A-class right of way. BRT could complete the running task according to schedule accurately, which possesses better passenger confidence and comfort.

The vehicles of BRT have high priority of right of way. On the basis of the BRT system, adopting TTS allows rapid buses have good grid effect, at the meantime greatly facilitate the transfer of passengers [10].

Case Study of TTS. In real life, because of limit of the bus company operating economic demands, the line coverage and travel demand factors, very few bus lines of turnaround time can be controlled within $1 \mathrm{~h}$ (60 min), while many bus routes one-way travel time could reach 50 min or more than $1 \mathrm{~h}$. And the application of bus rapid transit system in domestic also is not very wide, it is not need the use of TTS system optimization in the BRT system. In urban and rural transit, in spite of less sites and running unobstructed, due to the urban and rural bus still belonging to the regular bus, low vehicle running speed, and the long distance, the operation of the urban and rural bus turnaround time is usually long and accurate in time. Therefore, in this case, expand the time cycle of TTS to $2 \mathrm{~h}$ (120min) and used in the urban and rural bus lines.

In this paper, the data comes from Yongkang city bus lines between urban and rural areas. The urban and rural bus lines concentrated in Yongkang East Bus Station, suitable for using the TTS system optimization transfer service. Table 1 shows the minimum turnaround time and maximum acceptable departure interval of the lines. The data is calculated by the line distance and the vehicle average operating speed.

Table 1 the running time of the urban and rural bus line in Yongkang

\begin{tabular}{lcc}
\hline \multicolumn{1}{c}{ Line } & $\begin{array}{c}\text { Minimum Turnaround } \\
\text { Time (min) }\end{array}$ & $\begin{array}{c}\text { Maximum Acceptable } \\
\text { Departure Interval (min) }\end{array}$ \\
\hline A Line Tangxi & 118 & 35 \\
B Line Xixi & 97 & 25 \\
C Line Youchuan & 45 & 30 \\
D Line Zhiying & 49 & 24 \\
E Line Jinchuan & 75 & 60 \\
F Line Houtangnong & 70 & 54 \\
G Line Xiangzhu & 42 & 20 \\
H Line Tangxian & 66 & 14 \\
I Line Fangyan & 76 & 15 \\
J Line Xinlou & 102 & 30 \\
K Line Zhoushan & 55 & 28 \\
L Line Baiyun & 26 & 23 \\
M Line Yuxitou & 48 & 44 \\
N Line Qiancang & 38 & 12 \\
O Line Dachen & 56 & 22 \\
P Line Huanggang & 70 & 50 \\
Q Line Yongxiang & 64 & 30 \\
R Line Shuangshi & 104 & 60 \\
\hline
\end{tabular}

According to the design procedure of TTS network, if the basic pulse departure interval is $15 \mathrm{~min}$, the 18 bus lines need 69 vehicles in all, while the total difference between the single operating cycle and the minimum operating cycle is $209 \mathrm{~min}$, the total difference of single line shift operating cycle is $723 \mathrm{~min}$. When the basic pulse departure interval is $20 \mathrm{~min}$, the factors showed in Table 2 and 3. 
Table $2 \mathrm{~h}_{\mathrm{p}}=20$ min TTS bus line departure interval optimization (1)

\begin{tabular}{lcccccccccc}
\hline & \multicolumn{10}{c}{$\mathrm{h}_{\mathrm{p}}=20 \mathrm{~min}$} \\
\hline Line & $\mathrm{A}$ & $\mathrm{B}$ & $\mathrm{C}$ & $\mathrm{D}$ & $\mathrm{E}$ & $\mathrm{F}$ & $\mathrm{G}$ & $\mathrm{H}$ & $\mathrm{I}$ & $\Sigma$ \\
\cline { 2 - 10 }$T_{\min }$ & 118 & 97 & 45 & 49 & 75 & 70 & 42 & 66 & 76 & - \\
$\mathrm{h}$ : expected & 35 & 25 & 30 & 24 & 60 & 54 & 20 & 14 & 15 & - \\
$\quad$ provided & 20 & 20 & 20 & 20 & $40(60)$ & 40 & 20 & 10 & 10 & - \\
$T$ & 120 & 100 & 60 & 60 & 80 & 80 & 60 & 70 & 80 & - \\
$\Delta T=T-T_{\min }$ & 2 & 3 & 15 & 11 & 5 & 10 & 18 & 4 & 4 & 72 \\
$N$ & 6 & 5 & 3 & 3 & 2 & 2 & 3 & 7 & 8 & 39 \\
$N \bullet \Delta T$ & 12 & 15 & 45 & 33 & 10 & 20 & 54 & 28 & 32 & 249 \\
\hline
\end{tabular}

Table $3 \mathrm{~h}_{\mathrm{p}}=20$ min TTS bus line departure interval optimization (2)

\begin{tabular}{lcccccccccc}
\hline & \multicolumn{10}{c}{$\mathrm{h}_{\mathrm{p}}=20 \mathrm{~min}$} \\
\hline Line & $\mathrm{J}$ & $\mathrm{K}$ & $\mathrm{L}$ & $\mathrm{M}$ & $\mathrm{N}$ & $\mathrm{O}$ & $\mathrm{P}$ & $\mathrm{Q}$ & $\mathrm{R}$ & $\sum$ all \\
\cline { 2 - 10 }$T_{\min }$ & 102 & 55 & 26 & 48 & 38 & 56 & 70 & 64 & 104 & - \\
h: expected & 30 & 28 & 23 & 44 & 12 & 22 & 50 & 30 & 60 & - \\
$\quad$ provided & 20 & 20 & 20 & 40 & 10 & 20 & 40 & 20 & 60 & - \\
$T$ & 120 & 60 & 40 & 80 & 40 & 60 & 80 & 80 & 120 & - \\
$\Delta T=T-T_{\min }$ & 18 & 5 & 14 & 32 & 2 & 4 & 10 & 16 & 16 & 189 \\
$N$ & 6 & 3 & 2 & 2 & 4 & 3 & 2 & 4 & 2 & 67 \\
$N \bullet \Delta T$ & 108 & 15 & 28 & 64 & 8 & 12 & 20 & 64 & 32 & 600 \\
\hline
\end{tabular}

According to the tables, if the basic pulse departure interval is 20min, the 18 bus lines need 67 vehicles in all, while the total difference between the single operating cycle and the minimum operating cycle is $189 \mathrm{~min}$, the total difference of single line shift operating cycle is $600 \mathrm{~min}$. The data are all much better than the optimizing solution of $15 \mathrm{~min}$. When selecting pulse departure intervals for $20 \mathrm{~min}$, it is more economical for the bus company. It can meet the demand of passenger travel, both quick and convenient synchronous transfer service.

Establish the clock synchronous transfer system diagram is as follows: 


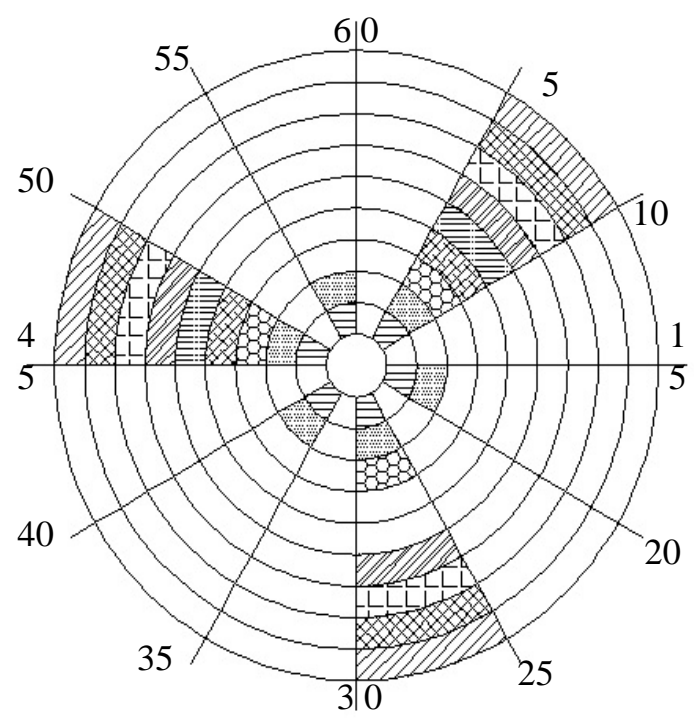

The First Hour

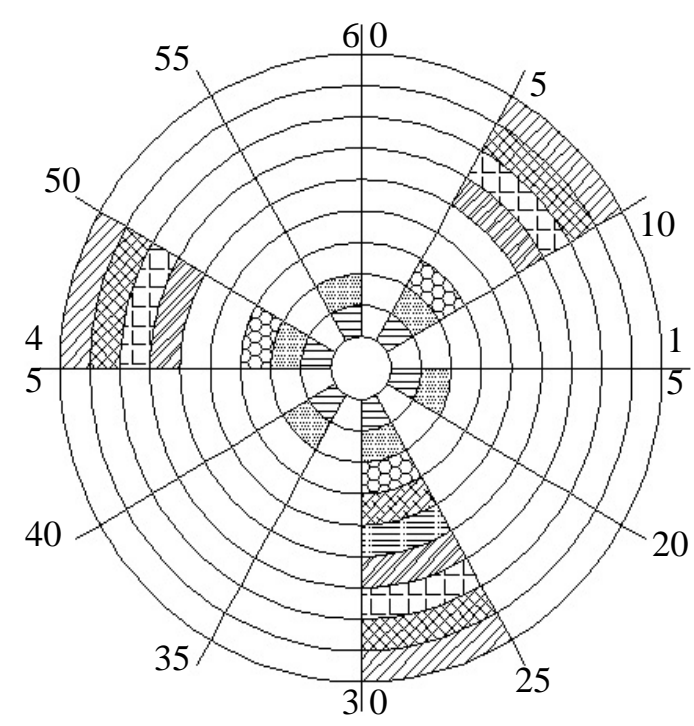

The Second Hour

From the outer to the inner track respectively Line $A \sim \mathrm{I}$ in $2 \mathrm{~h}$, shadow part is the period of five minutes before departure for passengers to get on the bus

Fig. 5 the $2 \mathrm{~h}$ cycle bus line departure clock diagram (1)
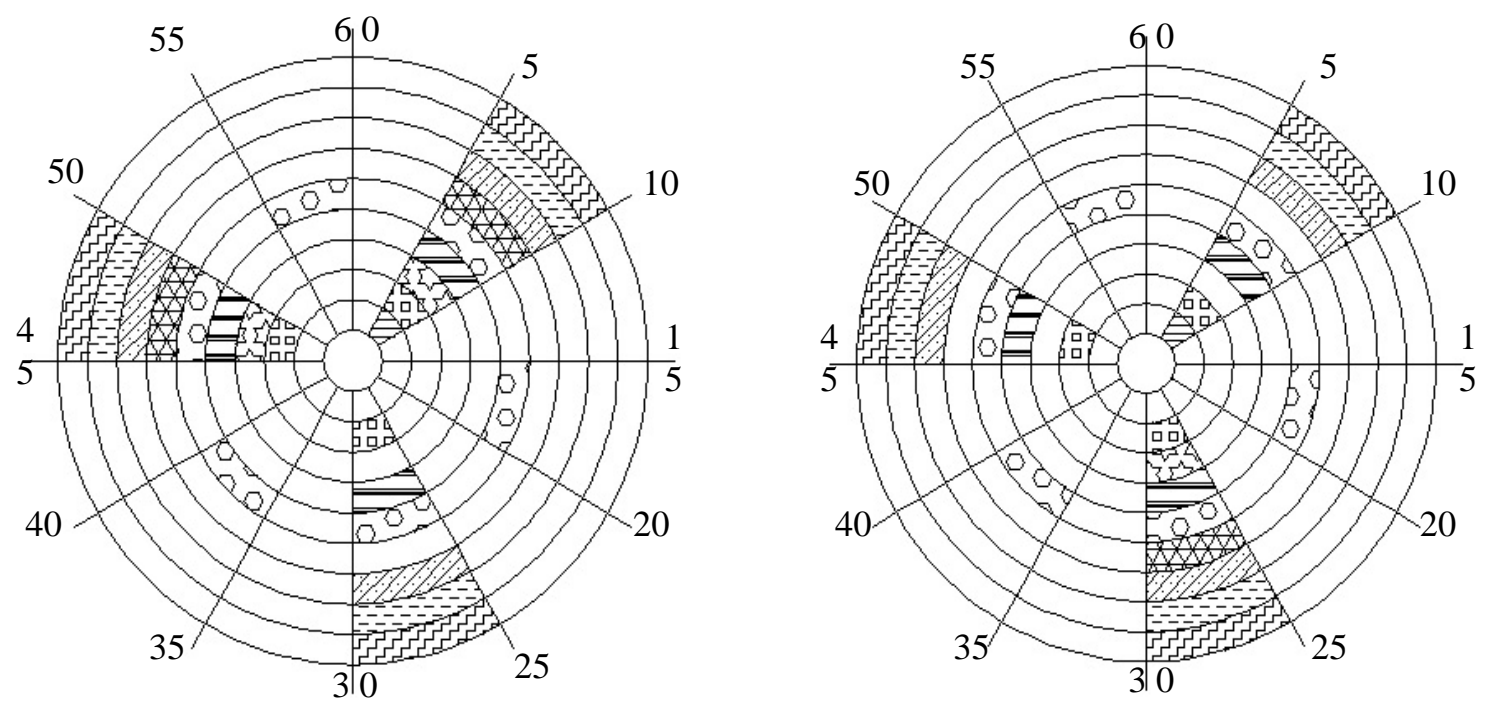

From the outer to the inner track respectively Line $\mathrm{J} \sim \mathrm{R}$ in $2 \mathrm{~h}$, shadow part is the period of five minutes before departure for passengers to get on the bus

Fig. 6 the $2 \mathrm{~h}$ cycle bus line departure clock diagram (2)

According to the figures, there are 3 lines' departure interval is $10 \mathrm{~min}$, which are $\mathrm{H}$ Line Tangxian, I Line Fangyan and N Line Qiancang. No matter from which line changing to these 3 lines can be very convenient. Moreover, there are 4 lines' departure interval is 40min, which are E Line Jinchuan, F Line Houtangnong, M Line Yuxitou and P Line Huanggang. Their departure time is at $10 \mathrm{~min}$ and $50 \mathrm{~min}$ in the first hour, and $30 \mathrm{~min}$ in the second hour. When a passenger wants to transfer to the four lines, the waiting time may be longer than others. Besides, the departure interval of R Line Huangshi is $60 \mathrm{~min}(1 \mathrm{~h})$. Passengers want to take this line should pay special attention to the travel time. The other 10 lines' departure intervals are all 20min after TTS optimization, that could provide convenient transfer service. 


\section{Summary}

On the basis of reading relevant literature and books, this paper treats the transfer barrier as an important factor to evaluate whether the transfer function is good enough. So in this paper, the factors that affect the transfer impedance are expounded and quantitative, and using examples to verify if the passengers spontaneously choose the line of smaller bus transfer barrier in travel. On the other hand, in the light of the problem of passenger transfer waiting time is too long, this paper focuses on the practical application of the TTS and gets the best bus departure schedule in the sample.

Due to the restriction of the author's academic level and the range of checking out, this paper has many deficiencies and shortcomings, and the study on transit service is also plain. But there is no denying the transfer service can greatly enhance the capacity of the public transport network. The transfer optimization service will also have a much broader application.

\section{References}

[1] Hurdle V F. Minimum cost locations for parallel public transit lines, J. Transportation Science, 1973, 7(4), 340-350.

[2] Kyte M, Stanley K, Gleason E. Planning, implementing, and evaluating a timed-transfer system in Portland, Oregon, J. Transportation Research Record, 1982(877), 23-29

[3] Ceder A. Bus timetables with even passenger loads as opposed to even headways, J. Transportation Research Record, 2001(1760), 3-9

[4] Tao Jiang. Heuristic transit network design method, D. ChengDu, Southwest Jiaotong University, 2006.

[5] Shuai Dai, Huaping Jiang, Haiyong Chen, Yanyan Chen. Public traffic transfer time reliability research, J. Journal of Highway and Transportation Research and Development, 2007, 24(9), 124-126,135.

[6] Jianxin Yan, Wenquan Li, Haijian Bai. Transit regional scheduling based on transfer optimization, J. Journal of Transportation Engineering and Information, 2010, 8(2), 81-86.

[7] Qin Shi, Yunmei Qin, Zhipeng Huang. The biggest timed transfer model on bus regional scheduling, J. China Journal of Highway and Transport, 2007, 20(6), 90-94.

[8] Vukan R.Vuchic. Urban transit: operations, planning, and economics, M. BeiJing, China Railway Publishing House, 2012.

[9] Xiaohong Jiang, Xiucheng Guo, Yiling Deng, Lichao Guo. Transfer system riding characteristics and its application in urban and rural public transportation network, J. Journal of Transportation Engineering and Information, 2014, 12(3), 58-63

[10] Yanbin Wang. Study on rapid transit waiting time scheduling based on TTS system, J. Communications Standardization, 2009, (11), 150-153 